\title{
Experimental comparison of a Gated-Viewing system and a 3-D Flash LADAR system in terms of range precision under different turbulence conditions
}

\author{
Göhler, Benjamin; Lutzmann, Peter \\ Fraunhofer Institute for Optronics, System Technologies and Image Exploitation IOSB \\ Gutleuthausstraße 1, 76275 Ettlingen, Germany
}

\begin{abstract}
For security and military applications, long-range automatic target recognition is a very important task. Therefore, in addition to a 2-D passive or active intensity image, 3-D information of a target is desirable. Besides a LADAR system, also a Gated-Viewing (GV) system can provide depth information by simply sliding the gate through the scenery. In this paper, the GV camera LIVAR ${ }^{\circledR} 500$ (Intevac, $640 \times 480$ pixels (binning mode), EBCMOS) is compared to a 3-D Flash LADAR camera (Advanced Scientific Concepts, $128 \times 128$ pixels, InGaAs APD) in terms of range precision. The sliding gates method requires several GV images (several laser pulses) with stepwise increased camera delay times. For the 3-D Flash LADAR camera, one laser pulse is sufficient because, for each pixel, the range is determined by the time-of-flight method. We have combined both cameras with the same pulsed laser illuminator with a wavelength of $1.57 \mu \mathrm{m}$. The maximal laser pulse energy was $67 \mathrm{~mJ}$. We have conducted field measurements at different times of day. Two reflectance panels and a vehicle at a distance of $2 \mathrm{~km}$ were recorded. The plates were positioned diagonal to the line of sight with an angle of about 45 degrees. They were used to determine the range precision, defined as the error standard deviation. Atmospheric turbulence, i.e. refractive index fluctuation along the propagation path, affects the laser pulse resulting in a degraded intensity image. The laser scintillometer BLS 900 (Scintec) measured the refractive index structure parameter $\mathrm{C}_{n}^{2}$ along the propagation path. The comparison of the two cameras was carried out under different turbulence conditions.
\end{abstract}

\section{Introduction}

In general, Gated-Viewing (GV) systems consist of a pulsed laser illuminator and a GV camera. After the laser pulse is emitted, the GV camera waits a predefined delay time until the detector elements integrate all photons that arrive within a very short integration time. Only laser photons that arrive from the corresponding range gate are collected; the fore- and the background are gated out. For the 3-D Flash LADAR camera, each detector element measures independently the time of flight of the transmitted laser pulse within its field of view. The result is a range image from only one laser pulse. However, with a GV camera, a range image can also be generated by capturing several GV images with slightly increased gate delay times ("sliding gates « method, [1]-[5]). In the following, the Intevac GV camera LIVAR ${ }^{\circledR} 500$ is compared to the 3-D Flash LADAR camera of Advanced Scientific Concepts ([6]) in terms of range precision.

\section{Experimental Set-up}

In the original configuration, the 3-D Flash LADAR system consists of a 3-D camera and an own synchronized pulsed laser source with a wavelength of $1.57 \mu \mathrm{m}$ for time-of-flight measurements. The maximal laser pulse energy of this laser source is $15 \mathrm{~mJ}$. Previous field tests showed that the operating range is limited to approximately $1 \mathrm{~km}$ under good atmospheric conditions and high target reflectivity. In order to study the influence of strong turbulence on range accuracy, the maximal operating range was increased by disabling this laser source and synchronizing the 3-D Flash LADAR camera with a flash lamp pumped Nd:YAG Q-switched laser with a maximal pulse energy of $67 \mathrm{~mJ}$. It has also a wavelength of $1.57 \mu \mathrm{m}$ (OPO shifted) and a pulse width of $7.1 \mathrm{~ns}$. This laser was also synchronized with the GV camera LIVAR 500. Additionally, the Scintec Boundary Layer Scintillometer (BLS) 900 receiver was mounted next to both systems. At a distance of approximately $2 \mathrm{~km}$ the Scintec BLS900 transmitter, a vehicle and two reflectance panels with $99 \%$ reflectivity were positioned next to each other. The vehicle and the panels had an angle of about 45 degrees with respect to the line of sight. A scheme of the experimental set-up and a visual image of the targets seen from the sensor position are depicted in Figure 1. Before comparing different sensors it has to be ensured that the comparison is as fair as possible. Preferably all parameters should be the same and the systems should be operated under the same conditions. 

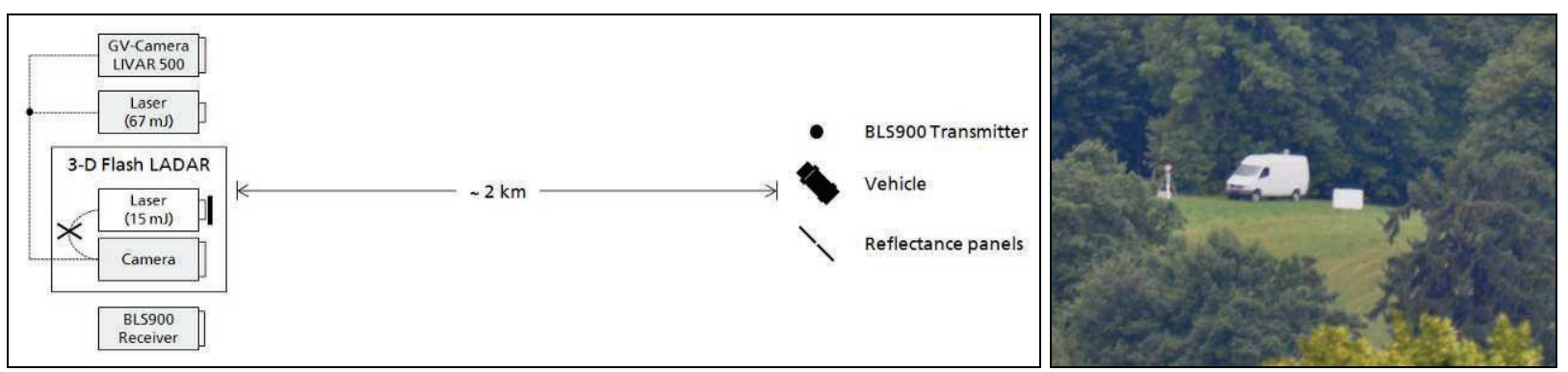

Figure 1. Left: Scheme of the experimental set-up and target positions at a distance of approximately $2 \mathrm{~km}$. The laser source with $67 \mathrm{~mJ}$ maximal pulse energy was synchronized with the GV camera LIVAR 500 as well as with the 3-D Flash LADAR camera. The laser of the 3-D Flash LADAR system was disabled. Right: Visual image of the targets seen from the sensor position.

Unfortunately this is not always possible. Here for instance, it is possible that either the field-of-views (FOVs) or the instantaneous field-of-views (IFOVs), i.e. the FOV of one detector element, of the two cameras have the same size by selecting appropriate optics. But due to the fact that the cameras have different resolutions, it is impossible to equal the FOVs and the IFOVs simultaneously. From Table 1 appears that the LIVAR 500 camera has a $5 \times 3.75$ times greater resolution and a 7.6 times smaller detector pixel size than the 3-D Flash LADAR camera. In order to have the same number of pixels on target, the IFOVs should be the same size. For this reason the GV camera was equipped with optics with a focal length of $250 \mathrm{~mm}$ and the 3-D Flash LADAR camera was equipped with a Cassegrain telescope with a focal length of $2032 \mathrm{~mm}$. Thus, the IFOVs of both cameras are approximately $50 \mu \mathrm{rad}$. For the IFOV calculations the small angle approximation IFOV $\approx$ (detector element size) / (optics focal length) was used. The drawback of this configuration is that the FOV of the GV camera is much greater than the angular target size and a lot of pixels of the GV camera are wasted.

\begin{tabular}{c|cc}
\hline Parameter & GV camera LIVAR 500 & 3-D Flash LADAR camera \\
\hline Resolution & $640 \times 480(2 \times 2$ binning mode $)$ & $128 \times 128$ \\
Detector pixel size & $13.4 \mu \mathrm{m}$ & $102.3 \mu \mathrm{m}$ \\
Optics focal length & $250 \mathrm{~mm}$ & $2032 \mathrm{~mm}$ \\
Aperture diameter & $12 \mathrm{~cm}$ & effective: $19.3 \mathrm{~cm}$ \\
Resulting IFOV & $\underline{53.6 \mu \mathrm{rad}}$ & $\underline{50.3 \mu \mathrm{rad}}$ \\
Resulting FOV & $34.3 \mathrm{mrad} \times 25.7 \mathrm{mrad}$ & $6.4 \mathrm{mrad} \times 6.4 \mathrm{mrad}$ \\
\hline
\end{tabular}

Table 1. Comparison of system parameter, selected optics and resulting FOVs of the two cameras.

The focal plane array of the GV-camera consists of $1280 \times 1024$ detector elements, each with a size of $(6.7 \mu \mathrm{m})^{2}$. For the measurements in this paper the camera was operated in a mode where $2 \times 2$ detector elements were binned together, resulting in a full resolution of $640 \times 512$ pixels, each with a size of $(13.4 \mu \mathrm{m})^{2}$, and then the standard resolution of $640 \times 480$ pixels was used. The detector element size of the 3-D Flash LADAR camera was calculated under the assumption of $100 \%$ fill factor from the fact that the FOV is 3 degree when the optics has a focal length of $250 \mathrm{~mm}$ (specification). The Cassegrain telescope has an aperture diameter of $20.3 \mathrm{~cm}$ and a central obscuration of $6.4 \mathrm{~cm}$ resulting in an effective aperture diameter of $19.3 \mathrm{~cm}$. Thus, the photon collecting area for the 3-D Flash LADAR camera is 2.6 times greater than for the GV-camera (12 cm aperture diameter). On the other hand the detector chip size of the 3-D Flash LADAR camera is 3.1 times greater than the detector chip size of the GV camera.

\section{Data}

With the different sensors described in Section 2, measurements were conducted. In Figure 2, a sample GV image of the target scene is depicted. The resolution is $640 \times 480$ pixels. The gate delay time was $12.995 \mu \mathrm{s}$ (corresponding to a range of $1949.25 \mathrm{~m}$ ) and the integration time was $385 \mathrm{~ns}$ (corresponding to a gate width of $57.75 \mathrm{~m}$ ). The rectangle defines the region of interest (ROI) that will be considered in the following analysis. The ROI contains the vehicle and the reflectance plates and has a size of $128 \times 128$ pixels. The BLS900 has periodically measured the refractive index structure parameter $\mathrm{C}_{\mathrm{n}}{ }^{2}$ at several times of day. In Figure 3 the $C_{n}{ }^{2}$ values are plotted versus the time of day. The times range from 10:15 AM until 2:31 PM and the measuring interval was 1 min. The measurements were conducted on a summer day. The turbulence strength was nearly constant $\left(\sim 10^{-14} \mathrm{~m}^{-2 / 3}\right)$ until approximately 1:30 PM 
because until midday it was heavily overcast. In the afternoon the sky was clearing and the turbulence strength rapidly increased $\left(\sim 7 \cdot 10^{-14} \mathrm{~m}^{-2 / 3}\right)$. The following two time intervals will be considered: 11:39 AM-11:45 AM for moderate

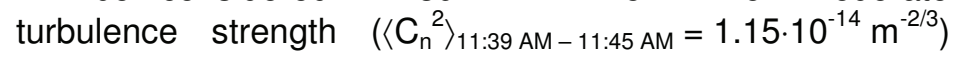
and 2:25 PM-2:31 PM for strong turbulence strength $\left(\left\langle\mathrm{C}_{\mathrm{n}}{ }^{2}\right\rangle_{2: 25 \mathrm{PM}-2: 31 \mathrm{PM}}=5.87 \cdot 10^{-14} \mathrm{~m}^{-2 / 3}\right)$. The average turbulence induced speckle size $d_{\mathrm{sp}}$ in the image plane can be calculated in the turbulence limited case $\left(\rho_{c}<\right.$ (optics aperture diameter)) with: $d_{\mathrm{sp}}=(1.22 \lambda \mathrm{f}) / \rho_{\mathrm{c}}$, where $\rho_{c} \approx\left(0.545(2 \pi / \lambda)^{2} C_{n}{ }^{2} R\right)^{-3 / 5}$ is the coherence radius for a spherical wave, $\lambda[\mathrm{m}]$ is the laser wavelength, $f[\mathrm{~m}]$ is the optics focal length, $C_{n}^{2}\left[\mathrm{~m}^{-2 / 3}\right]$ is the refractive index structure parameter and $R[\mathrm{~m}]$ is the distance between the sensors and the targets. With $\mathrm{R}=2 \mathrm{~km}$ and $\lambda=1.57 \mu \mathrm{m}$, the ratios $d_{\mathrm{sp}}$ :(detector pixel size) are nearly the same for the two

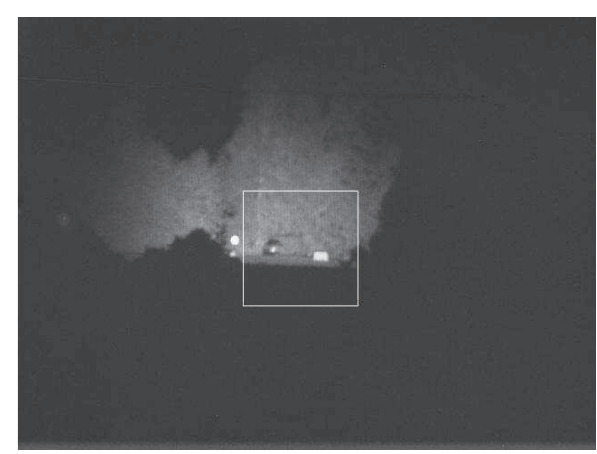

Figure 2. GV image with a gate position of $1949.25 \mathrm{~m}$ and a gate width of $57.75 \mathrm{~m}$. The rectangle indicates the $128 \times 128 \mathrm{ROI}$ that will be considered for GV imagery. cameras: $0.9: 1$ and 2.4:1 for the moderate and strong turbulence case, respectively. Thus, the speckle averaging behaviour of the two detectors is comparable in both turbulence conditions.

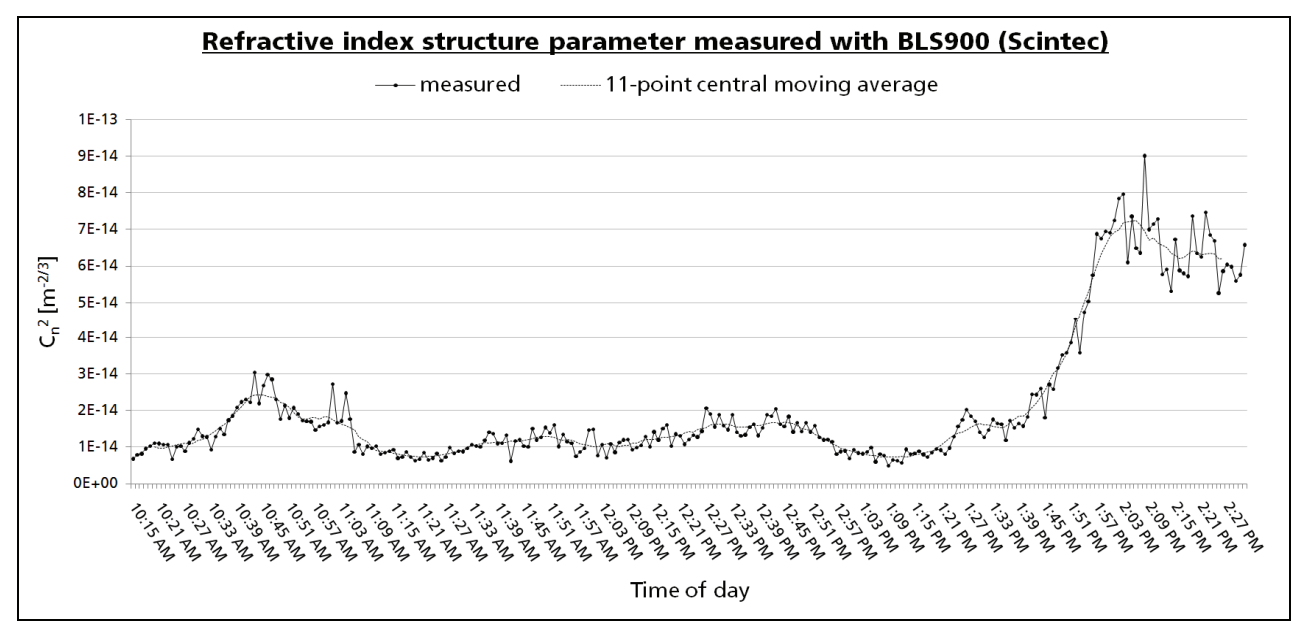

Figure 3. Solid line: Refractive index structure parameter $C_{n}{ }^{2}$ measured with the BLS 900 between 10:15 AM and 2:31 PM. The measuring interval was 1 min. Dashed Line: 11-point central moving average of the measured data.

\subsection{Moderate turbulence strength}

At 11:39 AM a range-scan with the GV camera LIVAR 500 was started. The gate delay times were successively increased from $12.995 \mu$ s to $13.185 \mu$ s with a step size of $5 \mathrm{~ns}$ (corresponding to gate positions from $1949.25 \mathrm{~m}$ to $1978.5 \mathrm{~m}$ with a step size of $0.75 \mathrm{~m}$ ). For each gate delay time, 25 frames were captured with a frame rate of $3 \mathrm{~Hz}$. The integration time was set to $195 \mathrm{~ns}$ (corresponding to a gate width of $29.25 \mathrm{~m}$ ). The range-scan was finished at 11:45 AM. In this period the average refractive index

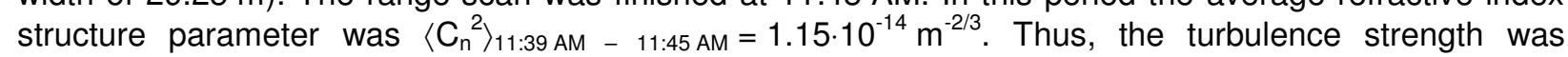
moderate. For each gate delay time, only the first captured image was processed, the following 24 images were skipped. In Figure 4 the resulting sequence is depicted line by line from left to right. In the upper left images the gate was still in front of the targets. In the upper right images the reflectance panels appeared before in the second and third line also the vehicle showed up. Finally, in the last line the silhouettes of the targets can be seen. During this GV range-scan, the 3-D Flash LADAR camera was also enabled at 11:41 AM and has simultaneously recorded 108 frames. Both cameras were synchronized to the same laser pulses. For further analysis of the 3-D Flash LADAR camera, only the fifth captured frame will be considered, the other 107 frames will be ignored. Due to higher sensitivity, the camera was operated in the so-called SULAR mode. In this mode the backscattered laser pulse is sampled at a predefined time in all pixels simultaneously (instead of independently in each pixel). The sampling interval is $2.34 \mathrm{~ns}$ and 20 samples are measured. By displaying the intensity values at a certain sampling time, one obtains images very similar to GV images. Using the nomenclature of GV systems, the gate width is $\sim 2 \mathrm{~m}$ (depending on laser pulse width) and the difference between two successive gate positions is $0.35 \mathrm{~m}$. In Figure 5 the sequence of the 20 slices is depicted line by line from left to right. 


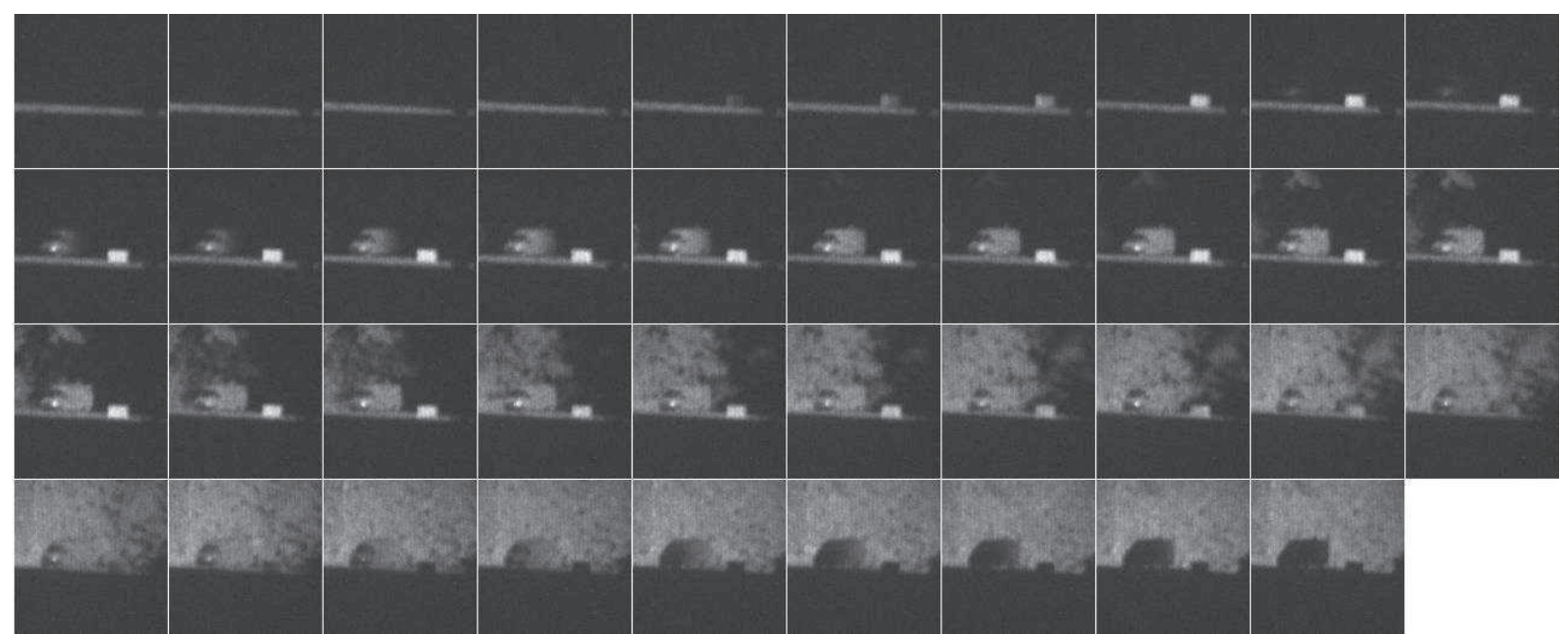

Figure 4. Sequence of $39 \mathrm{GV}$-images for moderate turbulence strength. The gate position was successively increased from $1949.25 \mathrm{~m}$ to $1978.5 \mathrm{~m}$ with a step size of $0.75 \mathrm{~m}$ and the gate width was $29.25 \mathrm{~m}$. Each image shows just the $128 \times 128 \mathrm{ROI}$ defined in Figure 2.

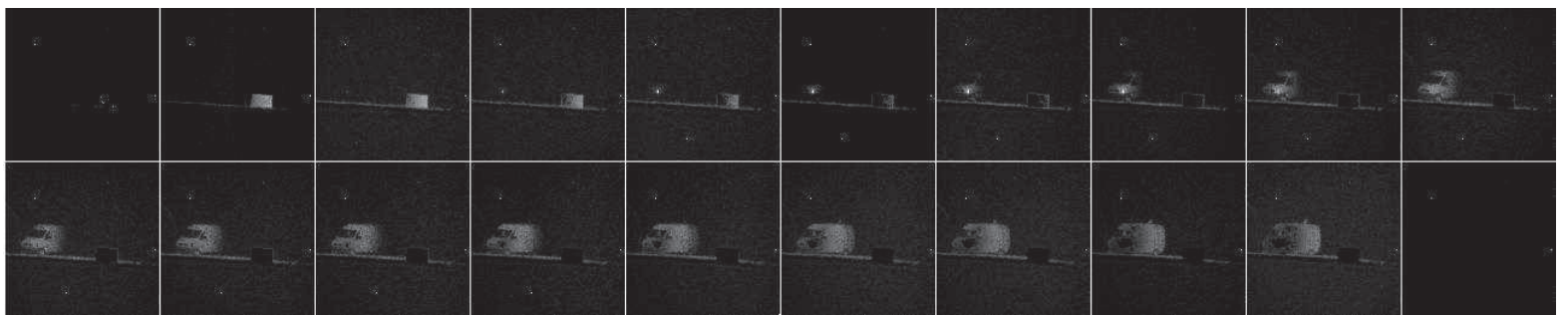

Figure 5. Sequence of the 20 slices of the 3-D Flash LADAR camera for moderate turbulence strength. The difference between two gate positions is $0.35 \mathrm{~m}$ and the gate width is $\sim 2 \mathrm{~m}$.

\subsection{Strong turbulence strength}

At 2:25 PM the second range-scan with the GV camera LIVAR 500 was started. Again, the gate delay times were successively increased from $12.995 \mu$ s to $13.185 \mu$ s with a step size of 5 ns (corresponding to gate positions from $1949.25 \mathrm{~m}$ to $1978.5 \mathrm{~m}$ with a step size of $0.75 \mathrm{~m}$ ). For each gate delay time, 25 frames were captured with a frame rate of $3 \mathrm{~Hz}$. The integration time was set to $195 \mathrm{~ns}$ (corresponding to a gate width of $29.25 \mathrm{~m}$ ). The range-scan was finished at 2:31 PM. In this period the average refractive index structure parameter was $\left\langle\mathrm{C}_{n}{ }^{2}\right\rangle_{2: 25} \mathrm{PM}-2: 31 \mathrm{PM}=5.87 \cdot 10^{-14} \mathrm{~m}^{-2 / 3}$. Thus, the turbulence strength was strong. For each gate delay time, only the first captured image was processed, the following 24 images were skipped. In Figure 6 the resulting sequence is depicted line by line from left to right.The laser beam divergence was decreased compared to the measurements under moderate turbulence conditions from

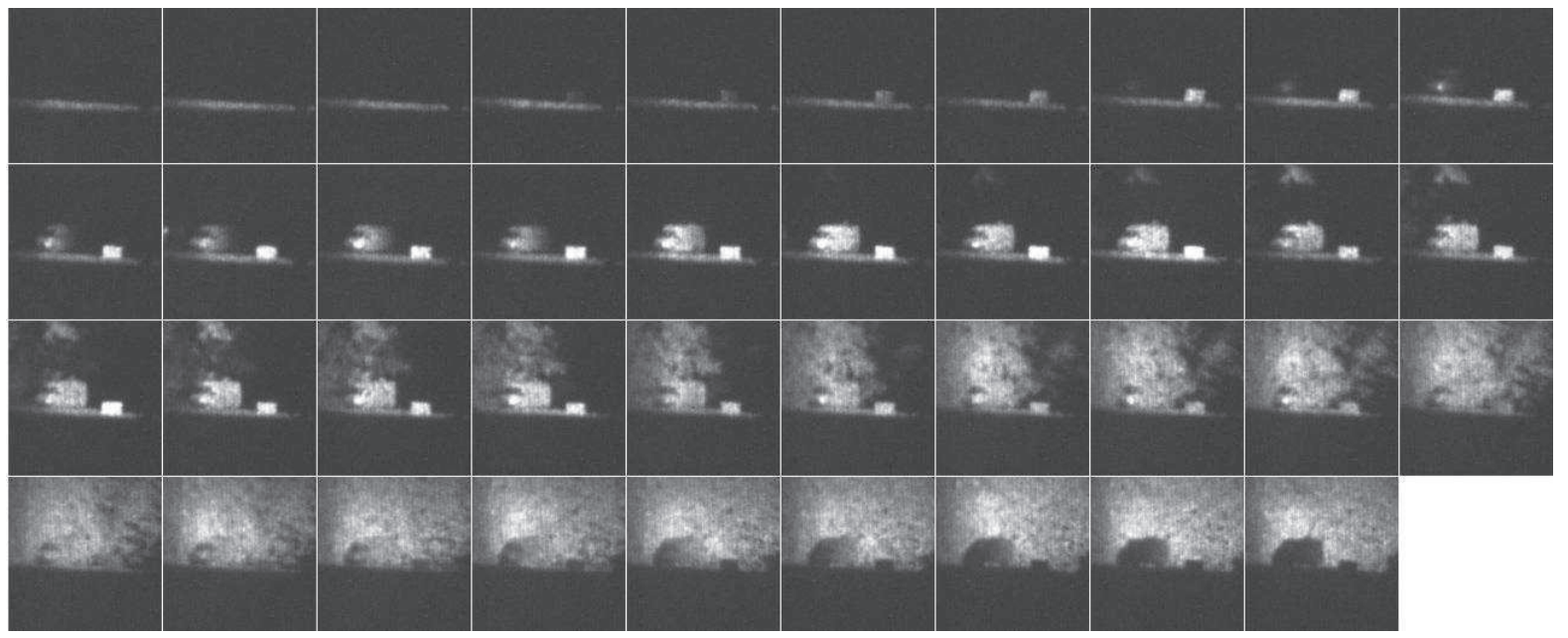

Figure 6. Sequence of 39 GV-images for strong turbulence strength. The gate position was successively increased from $1949.25 \mathrm{~m}$ to $1978.5 \mathrm{~m}$ with a step size of $0.75 \mathrm{~m}$ and the gate width was $29.25 \mathrm{~m}$. Each image shows just the $128 \times 128 \mathrm{ROI}$ defined in Figure 2. 
$\sim 19 \mathrm{mrad}$ to $\sim 12 \mathrm{mrad}$ resulting in higher intensities. However, the laser beam divergence is not changed during a range-scan. Again during this second GV range-scan, the 3-D Flash LADAR camera was also enabled at 2:27 PM and has simultaneously recorded 108 frames. Both cameras were synchronized to the same laser pulses. For further analysis of the 3-D Flash LADAR camera, only the fifth captured frame will be considered, the other 107 frames will be ignored. The camera was again operated in the SULAR mode with a sampling interval of $2.34 \mathrm{~ns}$. 20 samples were measured. In Figure 7 the sequence of the 20 slices is depicted line by line from left to right.

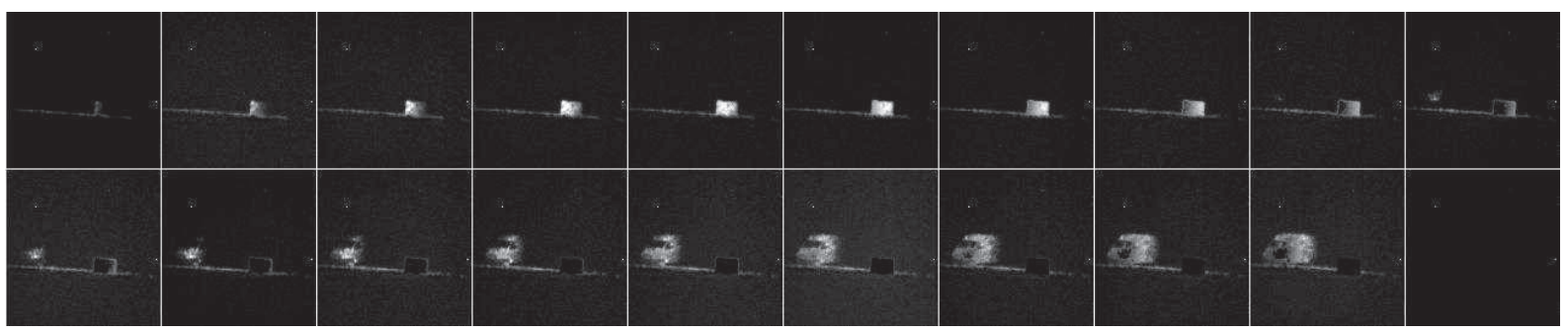

Figure 7. Sequence of the 20 slices of the 3-D Flash LADAR camera for strong turbulence strength. The difference between two gate positions is $0.35 \mathrm{~m}$ and the gate width is $\sim 2 \mathrm{~m}$.

\section{Methodology}

The four GV and 3-D Flash LADAR sequences from Section 3 are processed with the same algorithm to derive range values for each pixel. By plotting the intensity values of a pixel versus the gate delay time, one obtains the gate profile. The gate profile is approximated by the symmetric, piecewise polynomial function $\Pi_{p}$ in Figure 8 with $p=\left(p_{1}, p_{2}, p_{3}, p_{4}, p_{5}\right), p_{1} \leq p_{2} \leq p_{3}, p_{4} \leq p_{5}$.

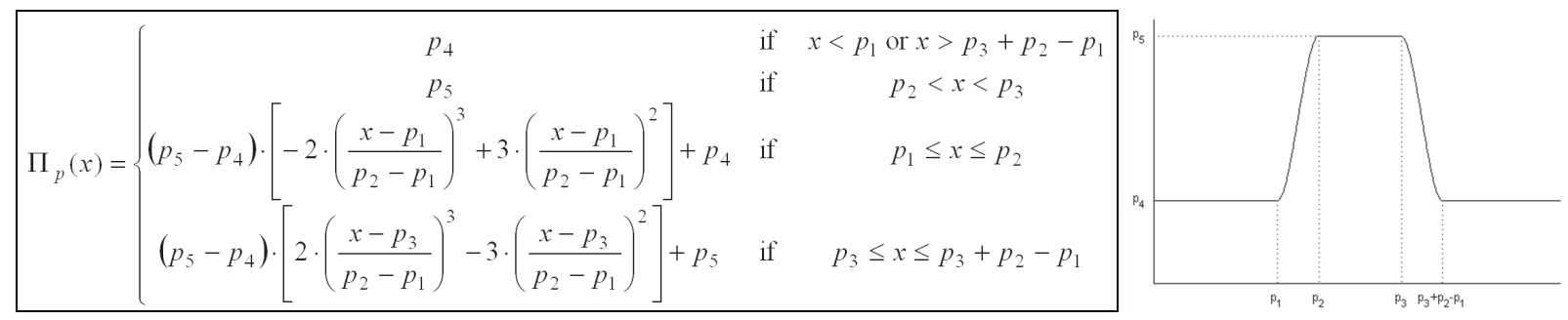

Figure 8. Parameterized symmetric function $\Pi_{p}$ with the parameters $p_{1}, \ldots, p_{5}$ as approximation of the gate profile function.

In the first step, this function is fitted to the measured intensity values for each pixel by the least squares method: Equation (1) in Table 2, where $g_{k}$ are the measured gray level values in a pixel $(k \in\{1,2, \ldots, n\})$ and $\mathrm{n}$ is the number of different gate delay times. As an example for this procedure, the measured gray level values and the corresponding fit for a pixel on the reflectance panel are depicted in Figure 9. The curve fitting is carried out for each pixel individually. From the optimal parameters $p_{2}$ and $p_{3}$, the range is derived by simply calculating their mean value: Equation (2) in Table 2. In the second step, the reflectance panel was utilized to determine the range precision. Therefore, a plane is fitted to the resulting point cloud of the reflectance panel. Let $R(x, y)$ be the calculated range array. $(x, y)$ denotes the horizontal

\begin{tabular}{|c|c|}
\hline Equation: & Number: \\
\hline $\min _{p} \sqrt{\sum_{\mathrm{k}=1}^{n}\left(\Pi_{p}(k)-g_{k}\right)^{2}}$ & (1) \\
\hline$R=\frac{p_{2}+p_{3}}{2}$ & (2) \\
\hline $\min _{a, b, c} \sqrt{\sum_{\mathrm{x}=1}^{m} \sum_{y=1}^{n}(P(x, y)-R(x, y))^{2}}$ & (3) \\
\hline$\sigma_{D}=\sqrt{\frac{1}{m \cdot n} \sum_{\mathrm{x}=1}^{m} \sum_{y=1}^{n}\left(D(x, y)-\mu_{D}\right)^{2}}$ & (4) \\
\hline$\mu_{D}=\frac{1}{m \cdot n} \sum_{\mathrm{x}=1}^{m} \sum_{y=1}^{n} D(x, y)$ & (5) \\
\hline
\end{tabular}

Table 2. Equations with equation numbers
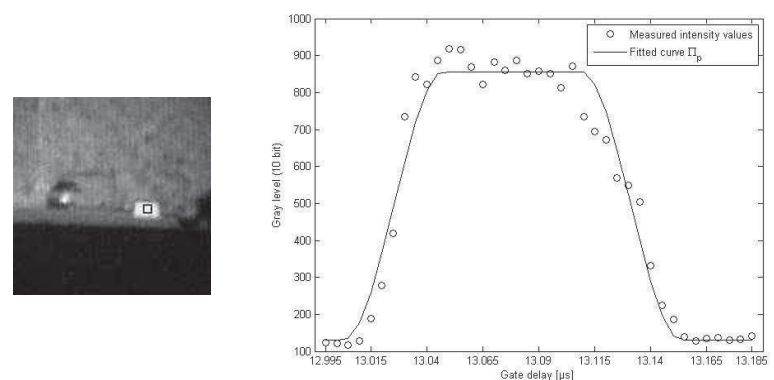

Figure 9. Left: GV image with square marker on reflectance panel. Right: Measured gray level values (circles) for the marked pixel versus the gate delay time and corresponding fit (solid line) of the function $\Pi_{p}$ from Figure 8. 
and vertical pixel number on the reflectance panel. The approach for the plane is $P(x, y)=a x+b y+c$, where the parameters $a, b$ and $c$ are again determined by the least squares method: Equation (3) in Table 2, where $m$ and $n$ are the number of pixels on the reflectance panel in the $x$ - and $y$-direction, respectively. Finally, the standard deviation $\sigma_{D}$ of the error array $D(x, y)=P(x, y)-R(x, y)$ is defined as range precision: Equation (4) in Table 2, where $\mu_{D}$ denotes the mean value of $D$ : Equation (5) in Table 2. The minimizations in Equations (1) and (3) in Table 2 were realized with the MATLAB ${ }^{\circledR}$ function fminsearch that uses a derivative-free simplex search method.

\section{Results}

The procedure from Section 4 is applied to the four GV and 3-D Flash LADAR sequences from Section 3. In Table 3, the range precision $\sigma_{D}$, calculated with Equations (4) and (5) are summarized for the two turbulence conditions and the two cameras. Unfortunately, no analysis was possible for the 3-D Flash LADAR camera under moderate turbulence conditions due to inapplicable data. Due to higher sensitivity, the 3-D Flash LADAR camera was operated in the SULAR mode. Unfortunately, the gate was set too far behind (see Figure 5). So, no 3-D reconstruction of the reflectance panel was possible in this case.

\begin{tabular}{|c|c|c|c|}
\hline \multicolumn{2}{|c|}{$\sigma_{D}$} & \multicolumn{2}{c|}{ Camera } \\
\cline { 3 - 4 } Turbulence & moderate & LIVAR 500 & 3-D Flash LADAR \\
\cline { 2 - 4 } & strong & $13.75 \mathrm{~cm}$ & - \\
\hline
\end{tabular}

Table 3. Summary of the range precision $\sigma_{D}$, calculated with Equations (4) and (5), for the two turbulence conditions and the two cameras.

The range precision $\sigma_{D}$ of the GV camera LIVAR 500 is degraded from $8.75 \mathrm{~cm}$ for moderate turbulence strength to $13.82 \mathrm{~cm}$ for strong turbulence strength. The range precision $\sigma_{D}$ of the 3-D Flash LADAR camera under strong turbulence conditions lies in between with a value of $10.04 \mathrm{~cm}$.

\section{Conclusions}

In summary, both the GV camera LIVAR 500 and the 3-D Flash LADAR camera exhibit comparable range precisions in the order of $10 \mathrm{~cm}$ for the presented procedure. While the 3-D Flash LADAR camera requires just one laser pulse to calculate a range image, the GV camera requires several laser pulses. Therefore, a GV camera is not suitable to calculate range images in dynamic scenarios (especially with target rotations) but in static ones. An advantage of the GV camera compared to the 3-D Flash LADAR camera is its high resolution of $640 \times 480$ pixels. Equipped with the telescope with $2032 \mathrm{~mm}$ focal length, a small IFOV of $6.6 \mu \mathrm{rad}$ can be achieved (Figure 10).

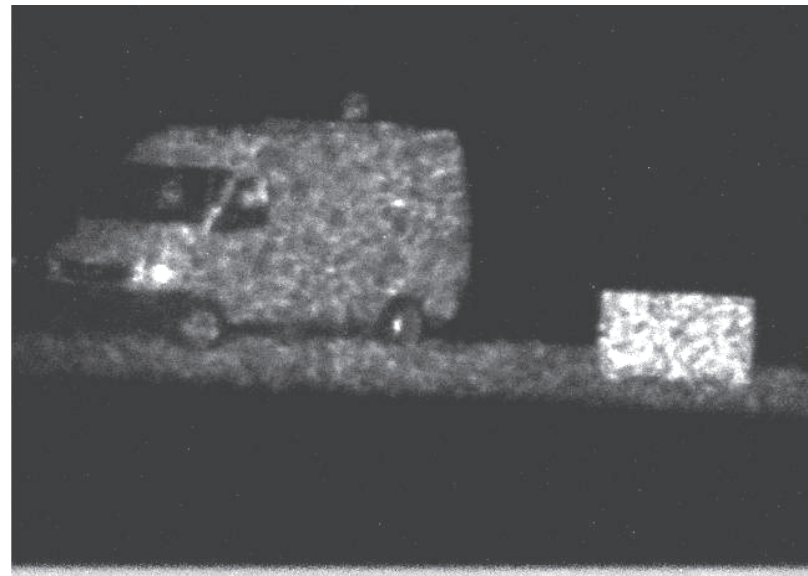

Figure 10. Small FOV GV image

\section{References}

[1] Repasi E., Lutzmann P., Steinvall O., Elmqvist M., Göhler B., Anstett G., "Advanced shortwavelength infrared range-gated imaging for ground applications in monostatic and bistatic configurations", Applied Optics Vol. 48, No. 31 (2009).

[2] Laurenzis M., "Evaluation metrics for range-gated active imaging systems using a Lissajous-type eye pattern", Applied Optics Vol. 49, No. 12 (2010).

[3] Bovenkamp E., Schutte K., "Laser Gated Viewing: an enabler for Automatic Target Recognition", Proc. SPIE 7684, $76840 Z$ (2010).

[4] Andersson P., "Long-range three-dimensional imaging using range-gated laser radar images", Optical Engineering Vol. 45 (3), 034301 (2006).

[5] Busck J., "Underwater 3-D optical imaging with a gated viewing laser radar", Optical Engineering Vol. 44 (11), 116001 (2005).

[6] Stettner R., Bailey H., Silverman S., "Large format time-of-flight focal plane detector development", Proc. SPIE 5791, 288-292 (2005). 